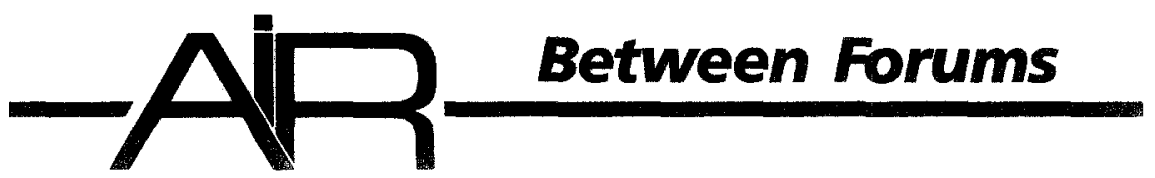

\title{
ON DEFINING COHERENCE AND INTEGRITY IN THE CURRICULUM
}

\author{
Joan S. Stark \\ National Center for Research to Improve Postsecondary Teaching and Learning \\ University of Michigan, Ann Arbor, Mi 48109
}

In the AAC report, Integrity in the College Curriculum: A Report to the Academic Community (1985), four terms are used frequently to convey a sense of desired excellence in postsecondary education. The terms are curricular coherence, curricular integration, curricular rigor and, lending the report its title, curricular integrity. Although the report's language implies meanings of these terms, its imprecision may lead to multiple interpretation by readers. Faculty who desire to implement curricular changes based on the $\mathrm{AAC}$ report, as well as institutional researchers who desire to assess such changes, will need to define these terms more precisely. It is my intent in this brief article to propose some useful definitions and to encourage others to disagree or to expand on these suggestions.

My argument begins with the useful notion that a curriculum is an academic plan. Both the AAC report and the earlier NIE report Involvement in Learning (1984) imply that curriculum may mean either a collection of course descriptions or, ex post facto, the intended or serendipitous experiences that students have while in college. Rather, in my judgment, curriculum is an academic plan, constructed by the faculty and possibly other knowledgeable individuals. The curriculum resembles a "trip tik" designed to lead to a desired destination. Like the planned trip itinerary, the curriculum or academic plan contains a number of elements appropriate to the outcomes to be achieved. Such elements include objectives specifying concepts, behaviors, skills, and attitudes to be learned, an explicit connection of the objectives to the needs of society and of individual learners, some evidence that the plan anticipates the prior experiences and knowledge of the students, a set of instructional techniques and learning aids believed to help learners achieve the objectives, and a specification of roles and obligations for the teacher and the learner. Thus the itinerary may vary as a function of different sets of students and teachers, although all are headed for a similar destination. Most importantly (but currently lacking in some 
academic plans), the curriculum must include a feedback mechanism which enables the planner to make itinerary adjustments based on the degree of success achieved by both teachers and learners.

This analogy may be carried one step further. A trip plan may exist, but no travelers may venture forth. Alternatively, those who venture forth may be detoured because of competing interests or because the plan inappropriately requires a different mode of transportation than what is offered. Without a sufficient number of travelers attempting the journey, the inappropriateness of the vehicle or unanticipated route obstacles may never be discovered and corrected. No feedback will be available to provide adjustments for the next traveler. Similarly, only by measuring the degree of success of various types of learners can the appropriateness of an academic plan be refined and improved.

Since the first term used in the AAC report, coherence, may be defined as a condition of logical consistency created by a set of well understood principles, an academic plan may be coherent although no students have pursued it. The plan expresses the purposes the faculty hope to achieve and their best judgement about what methods will achieve those objectives. Such purposes may reflect not only the views of the faculty as knowledge experts but their interpretations of societal needs, student needs, and educational strategies. In this sense, the typical course or program syllabus represents a coherent but incomplete academic plan or curriculum. Institutional researchers or program reviewers may examine academic plans for their degree of coherence. Even when appearing coherent, however, the plan may prove faulty for certain students or less than optimum for promoting learning of certain types of principles, behaviors, or attitudes. Thus, when complete, the plan will include a feedback link indicating how achievement of expectations may be measured.

The concept of integration adds a useful element to the curriculum equation. Integration means the incorporation and unification of elements into a whole. Thus curricular integration is a process occurring when students incorporate knowledge, behaviors, and attitudes included in the curricular plan into their own lives. A coherent curricular plan can exist without students, but curricular integration cannot take place until students interact with it. The degree to which integration takes place will depend upon student educational expectations (which may differ from those of the faculty) and cognitive and affective entry characteristics which influence how they organize knowledge and relate it to earlier learnings and experiences. It is at this point that student involvement (NIE, 1984) and the quality of student effort (Pace, 1983) enter the picture as partial determinants of integration. The logical consistency of the curricular plan is not automatically altered by the degree to which students are prepared, are involved, or exert effort. The 
degree to which curricular integration actually takes place, however, may be drastically altered by such factors. Thus, the attempts of students to successfully integrate knowledge via the academic plan complete the feedback link. Poor integration provides clues that faculty expectations of student success were in error and that the plan must be adjusted. It is not always easy to identify which factors are responsible for lack of curricular integration by students. In fact, even obvious clues are not always heeded; the plan may continue as before but curricular integration may be only partially achieved.

Academics are fond of speaking about curricular rigor and seem to use the term both to suggest that students should be challenged and that expectations should be established. While both of these goals are legitimate, rigor is a poor descriptor as applied to curriculum since it communicates inflexibility, stiffness, and inability to respond to stimuli. On the contrary, a view of curriculum as an academic plan with a feedback mechanism assures flexibility and potential response to clues which may help to improve student learning. If the term rigor was strictly interpreted, faculty proponents of a "rigorous" curriculum probably would either fail to include feedback mechanisms in their academic plans or would decline to make use of the information gathered. In my judgment, rigor is not a useful concept to convey the idea of curricular quality or excellence.

Integrity, implying a state of completeness and unity, is a far more effective curriculum concept than rigor. It is precisely to ensure the unification or completeness of the curricular plan that faculty should seek to assess the degree to which the learning expectations have been achieved. Assessment alone, of course, is insufficient to achieve unification. Faculty must also attempt to identify the possible obstacles and to adjust the plan to accommodate specific student needs. Just as the destination of the journey does not change, the expectations for learning need not necessarily be modified through curricular adjustment. Instead, other routes or other instructional vehicles may be found to be more effective for some students. Curricular integrity, then, implies not only the measurement of whether expectations set forth in the academic plan are achieved but the use of available information to improve the logical consistency or coherence of the plan.

Note that these definitions of curricular coherence, integration, and integrity resolve the time worn debate about whether extra-class activities are part of the curriculum. Such activities may be positively and purposefully included in the academic plan as specific ways of achieving expectations if they meet the test of coherence as, for example, in some residential learning plans. On the other hand, some things students learn out of class could not be included in the plan because they are inconsistent with its objectives. Additionally, while extra-class activities may enhance student ability to integrate learning, as in. concurrent learning in the work place, they may also 
hinder integration if, for instance, they reduce student effort or involvement. As with other student-based characteristics, extra-class activities that are recognized through feedback as aids or hindrances to achieving expectations will be dealt with when making adjustments in a curriculum with integrity. From this perspective, the lack of need to distinguish between in-class and extra-class activities effectively clarifies the heated debate concerning the recommendation of the NIE report that all students should spend considerable time on campus. In instances where time on campus would aid integration for the student, such requirements might be appropriate. In other instances, for example, when applying one's newly acquired learning in the work world aids integration, a different approach would be anticipated.

While most researchers would set aside the rhetoric of college catalogs as adequate measures of the curriculum, some maintain that college transcripts showing courses actually taken and passed are a reasonable representation of curriculum. Others assert that the curriculum has not been properly assessed unless actual student experiences are probed and analyzed. The definitions set forth here dictate that both measures are necessary but neither alone is sufficient. Consequently, to adequately represent the current state of the college curriculum at least four types of data must be examined: (1) the coherence of the academic plan as identified in detailed syllabi and course plans expressing expectations and modes; (2) the extent to which students meet the planned expectations; (3) the process through which the reasons why students are successful or unsuccessful are identified; and (4) the extent to which faculty use identified reasons for success to modify the academic plan.

Like the recent reports which provide recommendations for curricular change, the definitions provided here do not mandate particular content or particular instructional strategies for particular students. They do, however, reinforce the need to be explicit about the expectations higher education holds for students, and they provide a systematic framework through which the ambiguous concept of curriculum may be captured, studied, and improved.

\section{REFERENCES}

Association of American Colleges (1985). Integrity in the College Curriculum: A Report to the Academic Community. Wash., DC: AAC.

Pace, C. Robert (1983). Measuring the Quality of College Student Experiences. Los Angeles: University of California, Higher Education Research Institute.

Study Group on the Conditions of Excellence in American Higher Education (1984). Involvement in Learning: Realizing the Potential of American Higher Education. Wash., DC: National Institute of Education. 